

\title{
Ranking opportunity sets, indirect utility and indifferences*
}

\author{
Tom Potoms ${ }^{\dagger} \cdot$ Luc Lauwers ${ }^{\ddagger}$ \\ ${ }^{\dagger}$ Ecares programme (quantitative economics), \\ 42 Avenue Roosevelt, 1050 Brussels Belgium \\ ${ }^{\ddagger}$ Center for Economic Studies, Katholieke Universiteit Leuven, \\ Naamsestraat 69, 3000 Leuven, Belgium
}

September 4, 2012

\begin{abstract}
We characterize a new quasi-ordering on the collection of opportunity (or choice) sets. This new rule combines two criteria: the individual preferences on the universal set of options and the number of maximal options in the opportunity set. This new rule is compared with the indirect utility approach, and is further refined towards the leximax rule defined by Bossert, Pattanaik, and Xu (Journal of Economic Theory, 63, 1994).
\end{abstract}

JEL Classification Number: D71.

\section{Introduction}

Consider a finite set $X$ of alternatives and a complete and transitive (preference) relation on $X$. An opportunity set is a nonempty set of (available) alternatives. The literature on opportunity freedom tries to identify the value of opportunity sets. We refer to Barberà, Bossert and Pattanaik [1] and to Foster [4] for an overview. Let us recall two basic approaches: the cardinality approach interprets the cardinality of an opportunity set as a measure of freedom while the indirect utility approach evaluates an opportunity set by the value of its best element. Methods that take both components of well-being - quantity and quality of the alternatives - into account were proposed and characterized by Bossert, Pattanaik and $\mathrm{Xu}[2]$.

This note reconsiders the effect of indifferences between options when ranking opportunity sets. Let us take the example of an opportunity set that collects twelve equally good

*We thank Geert Dhaene, Johan Quaegebeur, and Luc Van Liedekerke for fruitful discussions. The authors are solely responsible for remaining shortcomings.

E-mail: Tom.Potoms@ulb.ac.be and luc.lauwers@econ.kuleuven.be. 
options, to which is added a stark thirteenth option, e.g. the option of being beheaded at dawn ${ }^{1}$. With respect to this extra option, Pattanaik and $\mathrm{Xu}$ [6, p. 179] write:

"We believe that even people who share the libertarian belief in the intrinsic value of freedom will agree that 'normally' this (option) would not constitute an increase in the agent's freedom."

This view criticizes the axiom of strict monotonicity according to which an opportunity set is ranked above its subsets. The cardinality rule and the four rules proposed by Bossert et al [2] - the leximax, the cardinality-first lexicographic, the preference-first lexicographic, and the dominance relation - all consider cardinality relevant and satisfy strict monotonicity. According to these rules, the addition of this thirteenth option increases the level of wellbeing. The indirect utility approach, on the other hand, proposes indifference between an opportunity set and one of its best options and, therefore, completely ignores the dimension of freedom in the well-being attached to the opportunity set.

We try to reconcile these concerns and propose the following rule. If the best alternative in opportunity set $A$ is better than the best alternative in opportunity set $B$, then $A$ represents a higher level of well-being than $B$. If the best alternatives are equally good, then we count the number of maximal options in both sets. The opportunity set with the highest number of maximal options represents the higher level of well-being. We label this rule as the indirect-utility-indifference rule. In the above example, a plausible ranking is obtained: the value attached to an opportunity set $(i)$ does not increase when this thirteenth option is made available, and $(i i)$ decreases when one of the twelve better options is deleted. In case the preference relation on $X$ is antisymmetric, then each indifference set is a singleton and this rule coincides with the indirect utility approach. Therefore, the indirect-utility-indifference rule refines the indirect utility approach only in the presence of indifferences. In real life, however, because the set $X$ of alternatives might be large, the preference relation of the individual might consider different options as (almost) equally good, which could cause (potentially large) indifference classes to appear. In such cases, the indirect-utility-indifference rule still maintains some degree of freedom alongside the individual preference relation. Moreover, the indirect-utility-indifference rule meets the above mentioned criticism: when a bad option becomes available the level of well-being does not increase. ${ }^{2}$

Our main result provides an axiomatization of the indirect-utility-indifference rule (Proposition 1). One of the axioms, which we label simple indirect preference, requires for each pair $x$ and $y$ of different options, that

$$
x \text { is equally good as } y \quad \Longrightarrow \quad\{x, y\} \text { is ranked above }\{y\} \text {. }
$$

\footnotetext{
${ }^{1}$ This example is used by Sen [8], who argues that we should take the preferences of the individual into account when assessing the freedom of an individual, see also Foster [3] and Puppe [7].

${ }^{2}$ Foster [4] introduces the $k$-censored freedom ranking, according to which opportunity sets with cardinality equal to or larger than $k$ are equally good: when the $k+1$ 'th option becomes available the level of well-being does not increase. In contrast, the level of censoring that occurs in the indirect-utilityindifference is controlled by the preference relation and depends upon the opportunity set.
} 
Information about how the preference relation ranks two options is used to rank two small opportunity sets. The indirect-utility-indifference rule obviously meets this axiom. The indirect utility method, on the other hand, satisfies the axiom of simple indirect indifference: for each pair $x$ and $y$ of different options, we have

$$
x \text { is equally good as } y \quad \Longrightarrow \quad\{x, y\} \text { is equally good as }\{y\} \text {. }
$$

As a matter of fact, the difference between the indirect-utility-indifference rule and the indirect utility approach boils down to the choice between these two axioms. Start from our axiomatization of the indirect-utility-indifference rule, replace the axiom of simple indirect preference by simple indirect indifference, then an axiomatization of the indirect utility method pops up. This extra result provides further support for the axioms that we use.

Section 2 introduces notation and axioms. Section 3 introduces the indirect-utilityindifference rule. Proposition 1 provides an axiomatization. Lemma 3 axiomatizes the indirect utility approach. Furthermore, we provide a link to a rule proposed by Pattanaik and $\mathrm{Xu}[6]$. We also show that the five axioms in Proposition 1 are independent. Section 4 weakens our position to restrict attention to the maximal options in an opportunity set. We introduce a rule that in addition to the maximal options also takes the second best options into account. As a matter of fact, each truncation of the leximax rule defines an ordering on the collection of opportunity sets.

\section{$2 \quad$ Notation and axioms}

The set $X$ of alternatives is finite. Let $R$ be a transitive and complete (hence, reflexive) binary relation on $X$. The asymmetric and the symmetric factors of $R$ are denoted by $P$ and $I$. A nonempty subset of $X$ is interpreted as a possible opportunity set that may be available to an individual equipped with the preference relation $R$. The collection of nonempty subsets of $X$ is denoted by $\Omega$. An option $a$ in the opportunity set $A$ is said to be $R$-maximal if $a R x$ for each $x$ in $A$. For each $A$ in $\Omega$, let $\max (A)$ denote the set of $R$-maximal elements in $A$. Since $X$ is finite, the set $\max (A)$ is nonempty. We abuse notation - recall that $R$ is defined on $X$ - and we use

$$
A R x \quad(\text { resp., } x R A),
$$

with $x$ in $X$ and $A$ in $\Omega$ as a shorthand for

$$
a R x \text { for each } a \text { in } A \text { (resp., } x R a \text { for each } a \text { in } A \text { ). }
$$

Let $\succsim$ be a transitive and reflexive binary relation on $\Omega$. The asymmetric and the symmetric factors of $\succsim$ are denoted by $\succ$ and $\sim$. We follow the axiomatic approach and define a relation $\succsim$ on $\Omega$ by means of a list of axioms or properties of the relation $\succsim$ on $\Omega$. We present and discuss eight axioms. 
Monotonicity. For each $A$ and $B$ in $\Omega$, we have $A \supseteq B$ implies $A \succsim B .^{3}$

If the opportunity set shrinks, then the level of well-being does not increase. Enlarging the choice set does not hurt the agent due to a more complex selection process. The restriction of this axiom to opportunity sets that have no more than two distinct elements is labeled as 'simple' monotonicity.

Simple monotonicity. For each $x$ and $y$ in $X$, we have $\{x, y\} \succsim\{x\}$.

Indifference. For each subset $A$ of $X$ and for each $x$ and $y$ in $X-A$, we have

$$
x I y \Longrightarrow A \cup\{x\} \sim A \cup\{y\} .
$$

Replace in an opportunity set an alternative with an alternative that is equally good. Then, the levels of well-being of the original and of the new opportunity set coincide. This axiom is related to 'neutrality' or 'anonymity': the particular content of an alternative is not important, only its ranking position matters. When the preference relation $R$ is linear -i.e., $x I y$ implies that $x$ and $y$ coincide - then this axiom is met in a trivial way.

Independence. For each $A$ and $B$ in $\Omega$ and for each $x$ in $X-(A \cup B)$, we have

$$
[\max (A) R x] \Longrightarrow[A \succsim B \Longleftrightarrow A \cup\{x\} \succsim B \cup\{x\}] .
$$

Consider two opportunity sets. The ranking between these two opportunity sets does not depend upon alternatives that are not preferred to the $R$-maximal alternatives of the better opportunity set. The addition (or removal) of such an alternative to (or from) both opportunity sets does not change their ranking. This axiom is related to the axiom of weak independence of Bossert et al [2].

The first lemma shows that the axiom of monotonicity is implied by the combination of simple monotonicity and independence.

Lemma 1. Let $R$ be a complete and transitive relation on $X$. Let the transitive and reflexive relation $\succsim$ on $\Omega$ satisfy Independence and Simple monotonicity. Then, $\succsim$ satisfies Monotonicity.

Proof. Let $A \supseteq B$. If $A=B$, then the reflexivity of $\succsim$ implies that $A \succsim B$. If $A \supset B$, let $a_{1} \in \max (A)$ and let $b_{1} \in \max (B)$. We distinguish two cases.

In case $a_{1} P b_{1}$, then $a_{1} \neq b_{1}$ and we use simple monotonicity to obtain $\left\{a_{1}, b_{1}\right\} \succsim\left\{b_{1}\right\}$. Write the options that are in $A$ and not in $B$ in $R$-decreasing order: $a_{1} R a_{2} R \cdots R a_{k}$. Use independence and obtain the following sequence of weak inequalities:

$$
\begin{array}{r}
\left\{a_{1}, a_{2}, b_{1}\right\} \succsim\left\{a_{2}, b_{1}\right\} \succsim\left\{b_{1}\right\}, \\
\left\{a_{1}, a_{2}, a_{3}, b_{1}\right\} \succsim\left\{a_{2}, a_{3}, b_{1}\right\} \succsim\left\{a_{3}, b_{1}\right\} \succsim\left\{b_{1}\right\}, \\
\left\{a_{1}, a_{2}, a_{3}, a_{4}, b_{1}\right\} \succsim\left\{a_{2}, a_{3}, a_{4}, b_{1}\right\} \succsim\left\{a_{3}, a_{4}, b_{1}\right\} \succsim\left\{a_{4}, b_{1}\right\} \succsim\left\{b_{1}\right\}, \\
\vdots \\
(A-B) \cup\left\{b_{1}\right\} \succsim \cdots \succsim\left\{a_{k-2}, a_{k-1}, a_{k}, b_{1}\right\} \succsim\left\{a_{k-1}, a_{k}, b_{1}\right\} \succsim\left\{a_{k}, b_{1}\right\} \succsim\left\{b_{1}\right\} .
\end{array}
$$

\footnotetext{
${ }^{3}$ If $B$ is a subset (resp. strict subset) of $A$, we write $A \supseteq B$ (resp. $A \supset B$ ).
} 
In each line, the final inequality follows from simple monotonicity. The transitivity of $\succsim$ implies that $(A-B) \cup\left\{b_{1}\right\} \succsim\left\{b_{1}\right\}$. Apply independence and add the options that are in $B-\left\{b_{1}\right\}$. Conclude that $A \succsim B$.

In case $a_{1} I b_{1}$, take $a_{1}=b_{1}$ and $a_{2} \in \max (A-B)$. Use simple monotonicity to obtain $\left\{a_{2}, b_{1}\right\} \succsim\left\{b_{1}\right\}$. Similar to the first case, repeated independence results in:

$$
\begin{array}{r}
\left\{a_{2}, a_{3}, b_{1}\right\} \succsim\left\{a_{3}, b_{1}\right\} \succsim\left\{b_{1}\right\}, \\
\left\{a_{2}, a_{3}, a_{4}, b_{1}\right\} \succsim\left\{a_{3}, a_{4}, b_{1}\right\} \succsim\left\{a_{4}, b_{1}\right\} \succsim\left\{b_{1}\right\}, \\
\left\{a_{2}, a_{3}, a_{4}, a_{5}, b_{1}\right\} \succsim\left\{a_{3}, a_{4}, a_{5}, b_{1}\right\} \succsim\left\{a_{4}, a_{5}, b_{1}\right\} \succsim\left\{a_{5}, b_{1}\right\} \succsim\left\{b_{1}\right\}, \\
\vdots \\
(A-B) \cup\left\{b_{1}\right\} \succsim \cdots \succsim\left\{a_{k-2}, a_{k-1}, a_{k}, b_{1}\right\} \succsim\left\{a_{k-1}, a_{k}, b_{1}\right\} \succsim\left\{a_{k}, b_{1}\right\} \succsim\left\{b_{1}\right\} .
\end{array}
$$

Again, it follows that $A \succsim B$.

Extension. For each $x$ and $y$ in $X$, we have $\{x\} \succsim\{y\}$ if and only if $x R y$.

Extension imposes that the ordering of the singleton opportunity sets reflects the ordering of the alternatives. This axiom is typical for rules that take indirect utility into account. In contrast, the quantity approach considers singleton opportunity sets as equally good (cf. Pattanaik and $\mathrm{Xu}[5]$ ).

Composition. For each $A$ and $B$ in $\Omega$ and for each $x$ in $X-(A \cup B)$, we have

$$
A \succsim B \text { and } \max (B) P x \quad \Longrightarrow \quad A \succsim B \cup\{x\}
$$

The ranking of two opportunity sets does not change when an option dominated by the worst-off set is added to this worst-off set. This axiom is related to the robustness axiom of Bossert et al [2] and ensures that a lack of indirect utility cannot be compensated by adding worse alternatives.

We show that the axiom of indifference is implied by simple monotonicity, extension, composition, and independence. Furthermore, the imposition of these four axioms partitions the collection $\Omega$. Each partition class collects the opportunity sets with the same indirect utility and the same number of maximal elements. These partition classes filter out the information - indirect utility and the number of maximal elements - that we consider relevant for the ranking of opportunity sets.

Lemma 2. Let $R$ be a complete and transitive relation on $X$. Let the transitive and reflexive relation $\succsim$ on $\Omega$ satisfy Simple monotonicity, Composition, Independence, and Extension. Then,

(i) for each $A$ and $B$ in $\Omega$, we have

$$
\max (A) I \max (B) \text { and }|\max (A)|=|\max (B)| \quad \Longrightarrow \quad A \sim B
$$

(ii) the relation $\succsim$ satisfies Indifference. 
Proof. Lemma 1 implies that $\succsim$ satisfies monotonicity. Let $A$ and $B$ be two opportunity sets that satisfy the premise of the first statement in the lemma. We proceed in three steps.

Step 1. Monotonicity, extension, composition, and independence imply that $A \sim \max (A)$. Proof. Since $A \supseteq \max (A)$, monotonicity implies $A \succsim \max (A)$. We now focus on the reverse inequality, $\max (A) \succsim A$. If $\max (A)$ happens to coincide with $A$, this inequality follows from the reflexivity of $\succsim$. Otherwise, let $x \in A-\max (A)$. Hence, for each $a$ in $\max (A)$ we have $a P x$ and, by extension, $\{a\} \succ\{x\}$. Monotonicity and transitivity imply $\max (A) \succ\{x\}$. Since $\max (A) \succsim \max (A)$, composition implies $\max (A) \succsim \max (A) \cup\{x\}$. In case $A-\max (A)$ contains an option $y$ different from $x$, then $\max (A) \succsim \max (A) \cup\{y\}$. Furthermore, independence implies

$$
\max (A) \cup\{y\} \succsim \max (A) \cup\{x\} \cup\{y\} .
$$

From transitivity it follows that $\max (A) \succsim \max (A) \cup\{x, y\}$. Repeat this argument for each $z$ in $A-\max (A)$ and obtain $\max (A) \succsim A$.

Step 2. Let $a_{1} I a_{2} I \ldots I a_{n}$. Opportunity sets with $k$ of these options are equally good.

Proof. If $k=1$, then extension implies that $\left\{a_{i}\right\} \sim\left\{a_{1}\right\}$ for each $i=2,3, \ldots, n$. Next, we consider $k=2$. Apply independence to $\left\{a_{1}\right\} \sim\left\{a_{2}\right\}$ and obtain that $\left\{a_{1}, a_{i}\right\} \sim\left\{a_{2}, a_{i}\right\}$ for each $i \notin\{1,2\}$. Apply independence to $\left\{a_{1}\right\} \sim\left\{a_{i}\right\}$ with $i \neq 1$ and obtain that $\left\{a_{1}, a_{j}\right\} \sim\left\{a_{i}, a_{j}\right\}$ with $j \notin\{1, i\}$. Conclude that each pair $\left\{a_{i}, a_{j}\right\}$ of options $(i \neq j)$ is equally good as $\left\{a_{1}, a_{2}\right\}$. Continue this argument to obtain the statement.

Step 3. Conclude that $A \sim B$ and that $\succsim$ satisfies indifference.

Proof. From Step 1 we learn that $A \sim \max (A)$ and $B \sim \max (B)$. Furthermore, all options in $\max (A) \cup \max (B)$ are equally good and $|\max (A)|=|\max (B)|$. Step 2 implies that $\max (A) \sim \max (B)$. Use transitivity of $\succsim$ to conclude that $A \sim B$.

Finally, consider statement $(i i)$. Obtain opportunity set $B$ by replacing options in $A$ with options that are equally good. It follows that $A$ and $B$ satisfy the premise of the first statement, and are equally good.

The next two axioms provide different answers to the question of how to rank a pair of equally good options against one single option.

Simple indirect preference. For each pair, $x$ and $y$, of different alternatives in $X$, we have

$$
x I y \Longrightarrow\{x, y\} \succ\{x\} .
$$

The level of well-being attached to a pair of equally good options is higher than the level of well-being attached to a singleton that selects one of these options. This axiom is weaker than the axiom of simple strict monotonicity proposed by Bossert et al [2] according to which each pair $\{x, y\}$ is strictly better than $\{x\}$. 
Simple indirect indifference. For each pair, $x$ and $y$, of different alternatives in $X$, we have

$$
x I y \Longrightarrow\{x, y\} \sim\{x\} .
$$

The level of well-being attached to a pair of equally good options is equal to the level of well-being attached to a singleton that selects one of these options.

\section{One option versus two equally good options}

We recall the indirect utility rule $\succsim_{I}$ and we introduce the indirect-utility-indifference rule, denoted by $\succsim_{I I}$. We provide an axiomatization of both rules which demonstrate how the difference between these two rules is captured by 'simple indirect indifference' and 'simple indirect preference'. We close this section with two remarks: we return to a rule proposed by Pattanaik and $\mathrm{Xu}[6]$ and we show the independency of the axioms used to characterize the indirect-utility-indifference rule.

Definition 1. Let $R$ be a complete and transitive relation on $X$. Let $\Omega$ be the collection of opportunity sets. The indirect utility rule, denoted by $\succsim_{I}$, is defined by

$$
A \succsim_{I} B \quad \text { if } \quad \max (A) R \max (B) .
$$

The indirect-utility-indifference rule, denoted by $\succsim_{I I}$, is defined by

$$
A \succsim_{I I} B \quad \text { if } \quad\left\{\begin{array}{l}
\max (A) P \max (B), \quad \text { or } \\
\max (A) I \max (B) \text { and } \quad|\max (A)| \geq|\max (B)| .
\end{array}\right.
$$

The relation $\succsim_{I I}$ keeps the strict ranking $\succ_{I}$ and refines the indifference relation $\sim_{I}$. Both rules satisfy monotonicity, extension, composition, and independence. Lemma 2 is the starting point in their characterizations.

Proposition 1. Let $R$ be a complete and transitive relation on the set $X$. There is only one transitive and reflexive relation $\succsim$ on $\Omega$ that satisfies Simple monotonicity, Composition, Extension, Independence, and Simple indirect preference. It is the indirect-utilityindifference rule.

Proof. It is easy to check that $\succsim_{I I}$ satisfies the axioms. Next, let $\succsim$ satisfy the axioms. We have to prove that $\succsim$ coincides with $\succsim_{I I}$. We proceed in different steps.

Step 1. The axioms of Lemma 2 (simple monotonicity, composition, independence, and extension) imply

$$
\max (A) P \max (B) \quad \Longrightarrow \quad A \succ B \text {. }
$$

Proof. Let $a \in \max (A)$. For each $b$ in $B$, we have $a P b$ and $\{a\} \succ\{b\}$ (use extension). In case $B=\{b\}$, we obtain $\{a\} \succ B$. Otherwise, let $b$ and $b^{\prime}$ be two different options in $B$. Use independence to obtain $\left\{a, b^{\prime}\right\} \succ\left\{b, b^{\prime}\right\}$. In combination with $\{a\} \sim\left\{a, b^{\prime}\right\}$ (use Lemma 2) it follows that $\{a\} \succ\left\{b, b^{\prime}\right\}$. Repeat this argument for each option in $B$ different 
from $b$ and $b^{\prime}$ and, again, conclude that $\{a\} \succ B$. Monotonicity implies $A \succsim\{a\}$. From the transitivity of $\succsim$ it follows that $A \succ B$.

Step 2. Add simple indirect preference to obtain

$$
\max (A) I \max (B) \text { and }|\max (A)|>|\max (B)| \quad \Longrightarrow \quad A \succ B \text {. }
$$

Proof. Let $A^{\prime}$ be a strict subset of $\max (A)$ with cardinality equal to $|\max (B)|$. Lemma 2 (ii) implies that $B \sim A^{\prime}$. Hence, it is sufficient to show that $A \succ A^{\prime}$. Now, according to the simple indirect preference principle we have $\{x, y\} \succ\{x\}$ with $x$ in $A^{\prime}$ and $y$ in $A-A^{\prime}$. Independence, simple monotonicity, monotonicity (Lemma 2), and transitivity imply that $A \succ A^{\prime}$.

Step 3. Conclusion.

Lemma 2 partitions the collection $\Omega$ into classes of opportunity sets that are equally good according to $\succsim_{I I}$. In order to complete the proof, it suffices to check whether these different partition classes are ordered in the 'right' way. This can be done by checking the following exhaustive list:

- $\max (A) P \max (B)$,

- $\max (A) I \max (B)$ and $|\max (A)|>|\max (B)|$,

- $\max (A) I \max (B)$ and $|\max (A)|=|\max (B)|$.

Step 1 (Step 2, Lemma 2) tackles the first (second, third) item. Conclude that the relation $\succsim$ coincides with $\succsim_{I I}$.

Let us now compare the indirect-utility-indifference rule and the indirect utility method. Lemma 3 shows that the difference between simple indirect preference and simple indirect indifference is able to capture the difference between these two rules. Indeed, replace in Proposition 1 the axiom of simple indirect preference by simple indirect indifference. The following characterization shows up.

Lemma 3. Let $R$ be a complete and transitive relation on $X$. There is only one transitive and reflexive relation on $\Omega$ that satisfies Simple monotonicity, Composition, Extension, Independence, and Simple indirect indifference. It is the indirect utility rule.

Proof. The indirect utility rule satisfies the five axioms. To show the converse, we first prove the following statement: for each $A$ in $\Omega$, we have

$$
a_{1} \in \max (A) \quad \Longrightarrow \quad A \sim\left\{a_{1}\right\} .
$$

Let $A=\left\{a_{1}, a_{2}, a_{3}, \ldots, a_{r}\right\}$ with $a_{1} R a_{i}$ for each $i=2,3, \ldots, r$. If $r=1$, the statement is immediate. In case $r>1$, then the following iteration holds. First, $\left\{a_{1}\right\} \sim\left\{a_{1}, a_{i}\right\}$ for each $i=2, \ldots, r$. Indeed, we have either $a_{1} I a_{i}$ or $a_{1} P a_{i}$. The indifference between $\left\{a_{1}\right\}$ 
and $\left\{a_{1}, a_{i}\right\}$ follows from either simple indirect indifference, or extension, composition, and simple monotonicity. Next, apply independence (add $a_{j}$ ) to obtain

$$
\left\{a_{1}, a_{i}, a_{j}\right\} \sim\left\{a_{1}, a_{j}\right\}, \quad \text { with } i, j=2,3, \ldots, r \text { and } i \neq j .
$$

From the transitivity of $\succsim$ it follows that $\left\{a_{1}\right\}$ is indifferent to each triple subset $\left\{a_{1}, a_{i}, a_{j}\right\}$ of $A$. By repeated application of independence we obtain that $\left\{a_{1}\right\}$ and $A$ are equally good. Now, use extension and conclude the lemma.

Lemma 3 is in line with Foster's [4] axiomatization of the indirect utility approach. In the above formulation of Lemma 3, the axiom of independence can be weakened to semi-independence:

Semi-independence. For each $A$ and $B$ in $\Omega$ and for each $x$ in $X-(A \cup B)$, we have

$$
[\max (A) R x] \Longrightarrow[A \succsim B \Longrightarrow A \cup\{x\} \succsim B \cup\{x\}] .
$$

Nevertheless, the above formulation of Lemma 3 is, for obvious reasons, intentional. Our characterizations of the indirect utility rule and the indirect-utility-indifference rule have four axioms in common: simple monotonicity, composition, extension, and independence. Let us impose these four axioms. The way in which a pair of equally good options is ranked against one of these options distinguishes the indirect utility rule from the indirectutility-indifference rules. In case the level of well-being attached to a pair of equally good options is considered higher than the level attached to one of these options, the indirectutility-indifference rule comes forward. In case such a pair is considered equally good as a singleton opportunity set, the indirect utility rule comes forward.

Remark 1. Pattanaik and Xu [6] define the 'maximal set' of an opportunity set $A$ as the collection of options in $A$ that are best in terms of some preference on $X$ that a reasonable person may have. They propose to rank opportunity sets on the basis of the cardinalities of their 'maximal sets'. This rule can be retrieved as follows. Denote by $\mathcal{P}$ the collection of the reasonable preferences and define an approval utility function $u: X \rightarrow\{0,1\}$ by $u(x)=1$ if option $x$ is $R$-maximal for at least one ordering $R$ in $\mathcal{P}$ and $u(x)=0$ otherwise. Then, the indirect-utility-indifference rule based upon this 'approval' preference relation (induced by $u$ ) coincides with the rule of Pattanaik and Xu.

Remark 2 (Independence of the five axioms). Let us list again our five axioms: simple monotonicity, composition, extension, independence, and simple indirect preference. For each axiom in this list we present a transitive and reflexive relation on $\Omega$ that violates this axiom and satisfies the other four axioms.

- Refine $\succsim_{I I}$ as follows:

$$
A \succsim B \quad \text { if } \quad\left\{\begin{array}{l}
A \succ_{I I} B \text { or, } \\
A \sim_{I I} B \text { and }|A-\max (A)| \leq|B-\max (B)| .
\end{array}\right.
$$

According to $\succsim$, non-maximal elements attach a negative effect to the opportunity set. The ranking rule $\succsim$ violates simple monotonicity: if $x P y$, then $\{x\} \succ\{x, y\}$. 
- The leximax rule (cf. the next section) ranks the pair $\{x, y\}$ with $x P y$ above $\{x\}$ and, hence, violates composition.

- Let $a$ and $b$ be two options. Let $R$ be a preference relation on $X$ such that each option in $X-\{a, b\}$ is preferred to $a$ and to $b$. Define a rule $\succsim$ on $\Omega$ as follows. The singletons $\{a\}$ and $\{b\}$ are not comparable. The remaining singletons are ordered according to $R$. Sets with two or more options are ordered according to $\succsim_{I I}$. This rule violates extension.

- Let $|X| \geq 3$. Restrict $\succsim_{I I}$ to the collection of singletons and pairs (hence, extension, simple monotonicity, and simple indirect preference are satisfied). Let $\{a, b\}$ be an

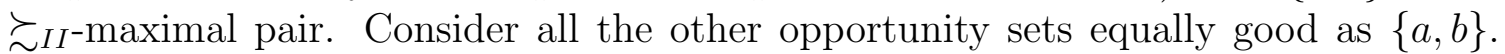
This rule violates independence.

- The indirect utility rule violates simple indirect preference.

\section{First best, second best, third best, ..., leximax}

The indirect-utility-indifference rule refines the indirect utility method as it considers the number of maximal options in an opportunity set as relevant. In this section, we develop a further refinement that also incorporates the second best options of an opportunity set. As such, more information is used and a finer ranking procedure is obtained.

Before we introduce this refinement, we recall the leximax rule (Bossert et al, 1994). Let $X$ be the finite set of options and let $R$ be a complete and transitive relation on $X$. Let $n=|X|$ be the number of different options in $X$. Let $u: X \rightarrow \mathbb{R}_{++}$be a representation of the preference relation $R$ on $X$. Each option obtains a positive utility. For each opportunity set $A=\left\{a_{1}, a_{2}, \ldots, a_{r}\right\}$ in $\Omega$, we define an $n$-vector

$$
u(A)=\left(u\left(a_{1}\right), u\left(a_{2}\right), \ldots, u\left(a_{r}\right), 0, \ldots, 0\right),
$$

that collects the utilities of the $r$ options in $A$, extended with $n-r$ zeros. The ordering $\succsim_{L}$ on $\Omega$ is based upon the leximax ordering $\geq_{L}$ on $\mathbb{R}^{n}$ : for each $A$ and $B$ in $\Omega$,

$$
A \succsim_{L} B \quad \text { if } \quad u(A) \geq_{L} u(B) .
$$

The ordering $\succsim_{L}$ does not depend upon the particular representation (provided $u(x)>0$ for each $x$ in $X$ ).

Let us slightly expand the notation. For each opportunity set $A$, the set $\max (A)$ collects the $R$-maximal options in $A$. The set $\max (A-\max (A))$ collects the second best options in $A$. In contrast to the set of maximal options, this collection of second best options might be empty. Indeed, if $\max (A)=A$, then $\max (A-\max (A))=\varnothing$. For each opportunity set $A$, let $\max _{2}(A)$ collect the first best and the second best options in the opportunity set $A$ :

$$
\max _{2}(A)=\max (A) \cup \max (A-\max (A)) .
$$


The set $\max _{2}(A)$ is not empty.

We now introduce the $\max _{2}$-rule, denoted by $\succsim_{2}$. This rule judges opportunity sets on the basis of, subsequently, the value of a first best option, the number of first best options, the value of a second best option, and the number of second best options. We use the leximax ordering $\succsim_{L}$ on $\Omega$ for its formal description. For each pair $A$ and $B$ of opportunity sets, we have

$$
A \succsim_{2} B \quad \text { if } \quad \max _{2}(A) \succsim_{L} \max _{2}(B) .
$$

In the same spirit, one can define the $\max _{k}$-rule that takes the first, second, third, ..., and $k^{\text {th }}$-best options into account. The $\max _{1}$-rule corresponds to the indirect-utility-indifference rule; the $\max _{n}$-rule to the leximax rule. The following axioms of robustness and independence are tailored to characterize the $\max _{2}$-rule.

Robustness $_{2}$. For each $A$ and $B$ in $\Omega$ and for each $x$ in $A$, we have

$$
A \succsim B \text { and } \max _{2}(A) P x \quad \Longrightarrow \quad A-\{x\} \succsim B .
$$

Options that are dominated by $\max _{2}(A)$ do not matter when ranking $A$ against $B$. Apply this axiom with $B=A$ and obtain that $\max _{2}(A) \succsim A$.

The $\max _{2}$-rule does not satisfy independence. Consider four options that satisfy $a P b P c P d$. Then $\{a, c\} \succ_{2}\{a, d\}$, while $\{a, b, c\} \sim_{2}\{a, b, d\}$. The entrance of option $b$ destroys the decisive role of options $c$ and $d$. The $\max _{2}$-rule satisfies the following modification.

Independence $_{2}$. For each $A$ and $B$ in $\Omega$ and for each $x$ in $X-(A \cup B)$, we have

$$
\left[\max _{2}(A) R x\right] \Longrightarrow[A \succsim B \Longleftrightarrow A \cup\{x\} \succsim B \cup\{x\}] .
$$

We also introduce the axioms of simple strict monotonicity (Bossert et al, 1994) and simple indifference.

Simple strict monotonicity. For each $x$ and $y$ in $X$, we have $\{x, y\} \succ\{x\}$.

Simple indifference ${ }_{2}$. For each $k$ in $\mathbb{N}$ and for each list, $x, y, z_{1}, z_{2}, \ldots, z_{2 k}$, of alternatives in $X$, with $z_{1} I z_{2} I \cdots I z_{2 k}$, we have

$$
\{x\} \succsim\{y\} \quad \Longleftrightarrow \quad\left\{x, z_{1}, z_{2}, \ldots, z_{k}\right\} \succsim\left\{y, z_{k+1}, z_{k+2}, \ldots, z_{2 k}\right\} .
$$

As the options $z_{i}$ are all equally good, there are at most two utility levels involved in each opportunity set. Therefore, we refer to this axiom as 'simple', although the opportunity sets might contain more than two options. If two equally large opportunity sets select all options but one in the very same indifference class, then the opportunity sets are ranked according to the options outside this indifference class. This axiom is satisfied by the leximax rule, but not by the indirect utility method and the indirect-utility-indifference rule. 
Lemma 4. Let $R$ be a complete and transitive relation on the set $X$. There is only one transitive and reflexive relation $\succsim$ on $\Omega$ that satisfies Extension, Monotonicity, Simple strict monotonicity, Robustness ${ }_{2}$, Independence 2 , and Simple indifference. It is the $\max _{2}$-rule.

Proof. The $\max _{2}$-rule satisfies the six axioms. Next, let $\succsim$ satisfy the six axioms. We have to show that $\succsim$ coincides with $\succsim_{2}$. Robustness 2 and monotonicity imply that $A \sim \max _{2}(A)$. Consider two opportunity sets $A$ and $B$. We use the following notation:

$$
\begin{aligned}
& \max (A)=\left\{a_{1}, a_{2}, \ldots, a_{k}\right\}, \quad \max _{2}(A)-\max (A)=\left\{a_{1}^{\prime}, a_{2}^{\prime}, \ldots, a_{l}^{\prime}\right\}, \\
& \max (B)=\left\{b_{1}, b_{2}, \ldots, b_{r}\right\}, \quad \max _{2}(B)-\max (B)=\left\{b_{1}^{\prime}, b_{2}^{\prime}, \ldots, b_{s}^{\prime}\right\}
\end{aligned}
$$

Recall that $l$ and $s$ might be equal to zero. Now, assume that $\max _{2}(A) \succsim_{L} \max _{2}(B)$. In order to show that $A \succsim B$, we have to check five cases: (i) $a_{1} P b_{1}$; (ii) $a_{1} I b_{1}$ and $k>r$; (iii) $a_{1} I b_{1}$ and $k=r$ and $a_{1}^{\prime} P b_{1}^{\prime}$; (iv) $a_{1} I b_{1}$ and $k=r$ and $a_{1}^{\prime} I b_{1}^{\prime}$ and $l>s ;(v) a_{1} I b_{1}$ and $k=r$ and $a_{1}^{\prime} I b_{1}^{\prime}$ and $l=s$. In each case, the relation $\succsim$ should rank $A$ and $B$ in the right way.

We only tackle the third case: $a_{1} I b_{1}$ and $k=r$ and $a_{1}^{\prime} P b_{1}^{\prime}$ imply $A \succ B$.

Extension implies $\left\{a_{1}^{\prime}\right\} \succ\left\{b_{1}^{\prime}\right\}$. Apply simple indifference to obtain

$$
\max (A) \cup\left\{a_{1}^{\prime}\right\} \succ \max (B) \cup\left\{b_{1}^{\prime}\right\} .
$$

Now, use independence $e_{2}$ to add the options $b_{2}^{\prime}, b_{3}^{\prime}, \ldots, b_{s}^{\prime}$, use robustness $s_{2}$ and the transitivity of $\succsim$, and conclude

$$
\max (A) \cup\left\{a_{1}^{\prime}\right\} \succ \max _{2}(B) .
$$

Use monotonicity and transitivity to obtain that $\max _{2}(A) \succsim \max (A) \cup\left\{a_{1}^{\prime}\right\} \succ \max _{2}(B)$. The other cases are tackled in a similar way.

\section{References}

[1] S. Barberà, W. Bossert, P.K. Pattanaik, Ranking sets of objects, in: S. Barberà, P.J. Hammond, Ch. Seidl (Eds.), Handbook of Utility Theory, Vol 2, Kluwer Academic Publishers, 2004.

[2] W. Bossert, P.K. Pattanaik, Y. Xu, Ranking opportunity sets: an axiomatic approach, J. Econ. Theory 63 (1994) 326-345.

[3] J. Foster, Notes on effective freedom, Mimeo (1992).

[4] J. Foster, Freedom, opportunity, and well-being, in: K.J. Arrow, K. Suzumura (Eds.), Handbook of Social Choice and Welfare, Vol 2, Elsevier, 2011.

[5] P.K. Pattanaik, Y. Xu, On ranking opportunity sets in terms of freedom of choice, Recherches Economiques de Louvain 56 (1990) 383-390.

[6] P.K. Pattanaik, Y. Xu, On preference and freedom, Theory and Decision 44 (1998) 173-198.

[7] C. Puppe, An axiomatic approach to 'preference for freedom of choice', J. Econ. Theory 68 (1996) 174-199.

[8] A.K. Sen, Markets and freedoms, Oxford Economic Papers 45 (1993) 519-541. 
Copyright (c) 2012 @ the author(s). Discussion papers are in draft form. This discussion paper is distributed for purposes of comment and discussion only. It may not be reproduced without permission of the copyright holder. Copies of working papers are available from the author. 